\title{
Asymptotic solutions of the evolution equation for the polarized nucleon structure function $g_{2}\left(x, Q^{2}\right)$
}

\author{
A. Ali \\ Deutsches Elektronen-Synchrotron, DESY, W-2000 Hamburg, FRG
}

\author{
V.M. Braun ${ }^{1}$ \\ Institut für Theoretische Physik, Universität Heidelberg, Philosophenweg 16,W-6900 Heidelberg, FRG \\ and Lengingrad Nuclear Physics Institute, SU-188 350 Gatchina, USSR
}

and

G. Hiller

II. Institut für Theoretische Physik der Universität Hamburg, W-2000 Hamburg, FRG

Received 20 May 1991

\begin{abstract}
We show that quark operators of twist 3 contributing to the polarized nucleon structure function $g_{2}^{\text {tw. } 3}\left(x, Q^{2}\right)$ decouple from the evolution equation for the quark-gluon operators of the same twist in two important limits, $N_{\mathrm{c}} \rightarrow \infty$ and $n \rightarrow \infty\left(N_{\mathrm{c}}\right.$ is the number of colours and $n$ refers to the $n$th moment of $g_{2}$ ). The anomalous dimensions for the quark operators turn out to be always the lowest ones in the spectrum. Asymptotic behaviour of $g_{2}\left(x, Q^{2}\right)$ in the region $1-x \ll 1$ is derived. Results of an extensive numerical study of the spectrum of anomalous dimensions for QCD and for the $N_{\mathrm{c}} \rightarrow \infty$ cases are presented.
\end{abstract}

\section{Introduction}

Spin dependent structure functions of the nucleon have received a lot of theoretical interest lately. The experimental data on the polarized structure function $g_{1}(x)$ from the EM Collaboration [1] have initiated animated discussions on the role of strange quarks and gluons in the polarized nucleon [2]. The second polarized nucleon structure function $g_{2}(x)$ has not been measured so far. A novel feature of $g_{2}(x)$ is that, unlike the spin-averaged nucleon structure functions and $g_{1}(x)$, it involves contributions from the quark-gluon correlations even in the limit $Q^{2} \rightarrow \infty$. In the language of Wilson operator expansion these are attributed to contributions of the sequence of local operators of twist 3

$O_{3}^{\sigma \mu 1 \ldots \mu_{n}}=\mathrm{AS} \bar{q} \gamma_{\sigma} \gamma_{5} \nabla_{\mu 1} \ldots \nabla_{\mu_{n}} q$

where $\mathrm{S}$ denotes symmetrization over $\mu_{1} \ldots \mu_{n}$, A antisymmetrizes over $\sigma$ and $\mu_{1}$, and $q$ is the quark field operator. It is known that the contribution of the twist-2 operators to $g_{2}(x)$ can be expressed in a compact form [3]:

$g_{2}^{\text {tw. } 2}(x)=-g_{1}(x)+\int_{x}^{1} \frac{\mathrm{d} y}{y} g_{1}(y)$,

\footnotetext{
Alexander von Humboldt Fellow.
} 
so that measurement of the difference $g_{2}^{\mathrm{tw} .3}\left(x, Q^{2}\right)=g_{2}\left(x, Q^{2}\right)-g_{2}^{\mathrm{tw} .2}\left(x, Q^{2}\right)$ would provide a clear signal for the higher twist effect. In this paper we elucidate the dependence of $g_{2}^{1 w .3}\left(x, Q^{2}\right)$ on the momentum transfer $Q^{2}$. We recall that in the case of spin averaged distributions higher twist operators only give rise to power corrections in $1 / Q^{2}$ to the leading twist part. Hence, their dependence on $Q^{2}$ is a matter of academic interest, in view of the rather constrained region of $Q^{2}$ in which such effects can be observed. In polarized scattering this $Q^{2}$ dependence can be studied experimentally for much larger values of $Q^{2}$.

In earlier papers [4], a simple evolution equation for the moments of $g_{2}^{\text {tw.3 }}\left(x, Q^{2}\right)$ has been written

$$
\int_{0}^{1} \mathrm{~d} x x^{j} g_{2}^{1 \mathrm{w} \cdot 3}\left(x, Q^{2}\right)=\left(\frac{\alpha_{\mathrm{s}}\left(Q^{2}\right)}{\alpha_{\mathrm{s}}\left(\mu^{2}\right)}\right)^{\gamma / b} \int_{0}^{1} \mathrm{~d} x x^{j} g_{2}^{\mathrm{tw} \cdot 3}\left(x, \mu^{2}\right),
$$

and the anomalous dimensions have been calculated by evaluating the operator (1) on the free quark states. Subsequently, it has been realized [5], that the derivation in ref. [4] leading to eq. (3) is in error, because quark operators (1) get mixed with quark-gluon operators of the same twist and quantum numbers. A complete basis of operators has been suggested and the mixing matrix has been calculated in ref. [6]. Similar calculations have later been performed by several authors [7-9].

The most significant departure from the results of ref. [4] is that the number of contributing operators to the structure function $g_{2}^{\text {tw. } 3}$ turns out to be increasing with the $(x)$-moment. The expressions for $n=2$ and 4 exemplify this:

$$
\begin{aligned}
& \int_{0}^{1} \mathrm{~d} x x^{2} g_{2}^{\mathrm{tw} \cdot 3}\left(x, Q^{2}\right)=\left(\frac{\alpha_{\mathrm{s}}\left(Q^{2}\right)}{\alpha_{\mathrm{s}}\left(\mu^{2}\right)}\right)^{\gamma / 2 / b}\left\langle\left\langle O_{2}^{1}\left(\mu^{2}\right)\right\rangle\right\rangle,
\end{aligned}
$$

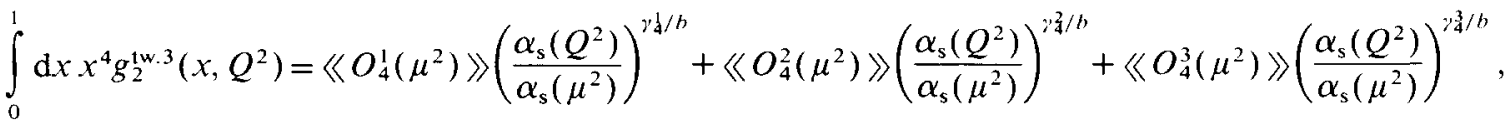

where $\left\langle\left\langle O_{j}^{i}\left(\mu^{2}\right)\right\rangle\right.$ are reduced matrix elements of the multiplicatively renormalized operators which are found by explicit diagonalization of the mixing matrix, $\gamma_{i}^{j}$ are the corresponding anomalous dimensions and $b=\left(11 N_{\mathrm{c}}-2 n_{\mathrm{f}}\right) / 3$, with $n_{\mathrm{f}}$ being the number of flavours. In the near future it will not be possible to distinguish between the terms with different anomalous dimensions experimentally.

Eq. (4) suggests that it is not possible to write down an evolution equation for $g_{2}^{1 \mathrm{w} .3}\left(x, Q^{2}\right)$ of the GribovLipatov-Altarelli-Parisi type [10], which would allow one to calculate the $Q^{2}$-dependence of the structure function from an assumed parametrization at some low virtuality. Hence unlike the spin averaged structure functions, one cannot use phenomenological models at lower $Q^{2}$ to predict $g_{2}^{\text {tw.3 }}\left(x, Q^{2}\right)$ at high momentum transfers. The anomalous dimensions $\gamma_{j}^{i}$ are not known analytically, which hinders analytic continuation to the small- $x$ region. For higher moments the number of independent operators increases and the situation becomes seemingly worse, see fig. 1 where the spectrum of anomalous dimensions of twist- 3 operators is shown for $n=2, \ldots$, 30. These difficulties compel us to search for some reasonable approximation to the exact solution, which could be used to analyse data when these become available.

We have found that eq. (3) provides actually an excellent approximate solution of the evolution equation and becomes exact in two important limits. The first is the limit of a large number of colours, $N_{\mathrm{c}} \rightarrow \infty$. The other one is the limit of a large number of the $(x)$-moment, $n \rightarrow \infty$, which governs the behaviour of the structure function $g_{2}\left(x, Q^{2}\right)$ in the region $1-x \ll 1$. To this end we solve explicitly the evolution equations for the quark-gluon operators obtained in ref. [6] and demonstrate that the particular combination of these operators which contributes to (1) diagonalizes the mixing matrix in the above mentioned limits. We present numerical calculations of the spectrum of anomalous dimensions for the flavour-singlet and -nonsinglet operators. Comparing these 


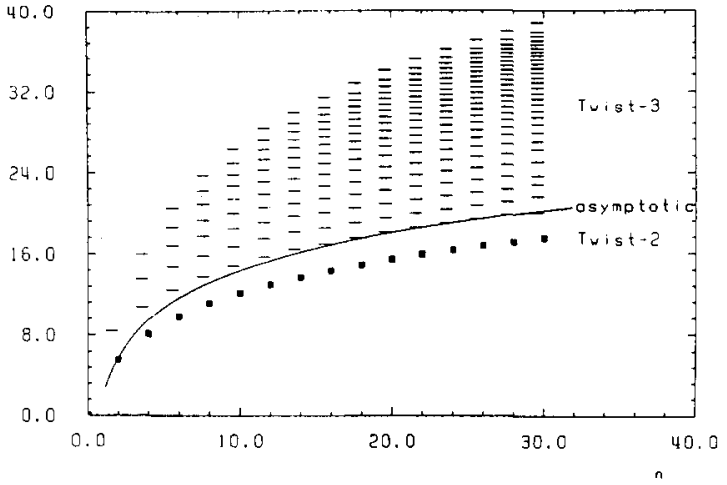

Fig. 1. Spectrum of anomalous dimensions of twist-3 nonsinglet operators compared to anomalous dimensions of twist- 2 operators (shown by solid squares) and to the asymptotic formula (22).

with our analytic solution for the large $N_{\mathrm{c}}$-limit, we find that the $1 / N_{\mathrm{c}}$ corrections are small. Thus, the quarkgluon operators couple only weakly to the structure function $g_{2}^{t w .3}$, which, however, does not justify the assumptions made in ref. [4], as the anomalous dimensions turn out to be different. Our results strongly suggest that anomalous dimensions of higher twist operators are analytic functions of the angular momentum.

\section{2. $Q^{2}$-evolution of $g_{2}\left(x, Q^{2}\right)$ in the $N_{\mathrm{c}} \rightarrow \infty$ limit}

We start by recalling that with the help of the equations of motion, the twist- 3 contributions to the polarized leptoproduction can be written in a number of equivalent ways. For our purpose it turns out to be convenient to use the particular form suggested in ref. [11]

$g_{2}(x)=-\tilde{g}_{2}(x)+\int_{x}^{1} \frac{\mathrm{d} y}{y} \tilde{g}_{2}(y)$,

with

$\tilde{g}_{2}(x)=g_{1}(x)-\frac{1}{4}[H(x)+H(-x)]-\frac{1}{2}[M(x)+M(-x)]$,

where the function $H(x)$ is determined by the nucleon matrix element of the twist-3 nonlocal operator:

$$
\begin{aligned}
& \sum_{q=\mathrm{u}, \mathrm{d} . \mathrm{s} . \ldots} e_{q}^{2} \int_{-1}^{1} \mathrm{~d} \xi\left\langle\mathrm{N}\left|g \bar{q}\left(\frac{1}{2} z\right)\left[\mathrm{i} \widetilde{G}_{\rho \nu}(\xi z)+\xi G_{\rho \nu}(\xi z) \gamma_{5}\right] z_{\nu} \neq q\left(-\frac{1}{2} z\right)\right| \mathrm{N}\right\rangle \\
& =2\left[(p z) s_{\rho}-(s z) p_{\rho}\right] \int_{-1}^{1} \mathrm{~d} x \exp (\mathrm{i} x p z) x H(x) .
\end{aligned}
$$

Here $p_{\rho}$ and $s_{\rho}=\bar{u}(p, s) \gamma_{\rho} \gamma_{s} u(p, s)$ are, respectively, the momentum and the spin-vector of the nucleon, and $z_{\mu}$ is a light-like vector. The gauge factors are not shown for brevity. We use the notations $\sigma_{\mu \nu}=\frac{1}{2} \mathrm{i}\left[\gamma_{\mu}, \gamma_{\nu}\right]$, $\tilde{G}_{\mu \nu}=\frac{1}{2} \epsilon_{\mu \nu \alpha \beta} G_{\alpha \beta}$, and $G_{\alpha \beta}$ is the QCD field-strength tensor. The covariant derivative reads as $\nabla_{\mu}=\partial / \partial x_{\mu}-\mathrm{i} g A_{\mu}$. For completeness we have included in (6) also the contribution of the quark mass operator, which is actually omitted in what follows:

$\sum_{q=\mathbf{u} . \mathrm{d} . \mathrm{s}, \ldots . .} e_{q}^{2}\left\langle\mathrm{~N}\left|\bar{q}\left(\frac{1}{2} z\right) \mathrm{i} m_{q} \sigma_{\rho \nu} z_{\nu} \gamma_{5} q\left(-\frac{1}{2} z\right)\right| \mathrm{N}\right\rangle=\left[(p z) s_{\rho}-(s z) p_{\rho}\right] \int_{-1}^{1} \mathrm{~d} x \exp (\mathrm{i} x p z) x M(x)$. 
Our strategy will be to work directly with the nonlocal operator contributing to ( 7 ) rather than to go over to the series of local operators. We start with the flavour nonsinglet case and define

$S_{\mu}^{ \pm}(\alpha, \beta, \gamma)=g \bar{q}(\alpha z)\left[\mathrm{i} \widetilde{G}_{\mu \nu}(\beta z) \pm G_{\mu \nu}(\beta z) \gamma_{s}\right] z_{\nu} \neq q(\gamma z)$.

The operators $S^{+}$and $S^{-}$do not mix under renormalization and the evolution equation for them is written in the form of a certain integral transform on the light-cone. The corresponding kernel $K^{ \pm}$is given by eq. (6.2) in ref. [8].

We further define

$S_{\mu}^{ \pm}(\alpha, \beta, \gamma)=\frac{1}{2 \pi \mathrm{i}} \int_{1 / 2-\mathrm{i} \infty}^{1 / 2+\mathrm{i} \infty} \mathrm{d} j(\alpha-\gamma)^{j-2} S_{\mu}^{ \pm}(j, \xi), \quad \xi=\frac{\alpha+\gamma-2 \beta}{\alpha-\gamma}$.

The complex angular momentum $j$ is a trivial invariant of the evolution equation. Combinings eqs. (7), (9), and (10) we obtain, up to trivial flavour factors,

$$
\begin{aligned}
& \frac{1}{2} \int_{-1}^{1} \mathrm{~d} \xi\left[(1-\xi)\left\langle\mathrm{N}\left|S_{\rho}^{+}(j, \xi)\right| \mathrm{N}\right\rangle+(1+\xi)\left\langle\mathrm{N}\left|S_{\rho}^{-}(j, \xi)\right| \mathrm{N}\right\rangle\right] \\
& \quad=2\left[(p z) s_{p}-(s z) p_{\rho}\right] \Gamma(-j+1)\left[\exp \left(-\frac{1}{2} \mathrm{i} \pi\right) p z\right]^{j-1} \int_{-1}^{1} \mathrm{~d} x(x+\mathrm{i} \epsilon)^{j} H(x) .
\end{aligned}
$$

Neglecting contributions to the evolution equation which are down by $1 / N_{\mathrm{c}}^{2}$ we obtain after some algebra

$$
S^{ \pm}(j, \xi)^{\mu_{2}^{2}}=S^{ \pm}(j, \xi)^{\mu_{1}^{2}}-\frac{\alpha_{\mathrm{s}}}{4 \pi} \ln \left(\frac{\mu_{2}^{2}}{\mu_{1}^{2}}\right) \int_{-1}^{1} \mathrm{~d} \eta K_{j}^{ \pm}(\xi, \eta) S^{ \pm}(j, \eta)^{\mu_{1}^{2}},
$$

where $\# 1$

$$
\begin{aligned}
& \frac{1}{N_{\mathrm{c}}} K_{j}^{ \pm}(\xi, \eta)=\frac{5}{2} \delta(\xi-\eta)-\frac{\theta(\xi-\eta)}{\xi-\eta} \frac{1+\eta}{1+\xi}\left[\frac{1+\eta}{1+\xi}+\left(\frac{1-\xi}{1-\eta}\right)^{j}\right]+\delta(\xi-\eta) \int_{-1}^{\xi} \frac{\mathrm{d} \eta^{\prime}}{\xi-\eta^{\prime}} \frac{1+\eta^{\prime}}{1+\xi}\left[1+\left(\frac{1-\xi}{1-\eta^{\prime}}\right)^{2}\right] \\
& \quad-\frac{\theta(\eta-\xi)}{\eta-\xi} \frac{1-\eta}{1-\xi}\left[\frac{1-\eta}{1-\xi}+\left(\frac{1+\xi}{1+\eta}\right)^{j}\right]+\delta(\eta-\xi) \int_{\xi}^{1} \frac{\mathrm{d} \eta^{\prime}}{\eta^{\prime}-\xi} \frac{1-\eta^{\prime}}{1-\xi}\left[1+\left(\frac{1+\xi}{1+\eta^{\prime}}\right)^{2}\right] \\
& \quad-\left(4 \frac{\theta(\xi-\eta)}{(1+\xi)^{3}}\left\{\frac{2}{j}\left[1-\left(\frac{1-\xi}{1-\eta}\right)^{j}\right]-\frac{1-\eta}{j+1}\left[1-\left(\frac{1-\xi}{1-\eta}\right)^{j+1}\right]\right\}\right)\left(K^{+}\right), \\
& \quad-\left(4 \frac{\theta(\eta-\xi)}{(1-\xi)^{3}}\left\{\frac{2}{j}\left[1-\left(\frac{1+\xi}{1+\eta}\right)^{j}\right]-\frac{1+\eta}{j+1}\left[1-\left(\frac{1+\xi}{1+\eta}\right)^{j+1}\right]\right\}\right)\left(K^{-}\right),
\end{aligned}
$$

The two expressions in large parentheses in the last line correspond to the contributions to $\mathrm{K}^{+}$and $\mathrm{K}^{-}$, respectively. To find the multiplicatively renormalized local operators one should consider the corresponding homogeneous equation for positive integers $j \geqslant 2$ :

$\int_{-1}^{1} \mathrm{~d} \eta K_{j}^{ \pm}(\xi, \eta) f_{j}(\eta)=\gamma_{j} f_{j}(\xi)$

\#1 The corresponding expression in ref. [8] contains misprints. 
Each solution of (14) in the form $f=\sum_{m+n=j-2} a_{m n}(1+\xi)^{m}(1-\xi)^{n}$ yields a renormalization group invariant local operator $\alpha_{\mathrm{s}}{ }^{-\gamma_{j} / b} \sum_{m+n=j-2} a_{m n} \bar{\psi}(\vec{\nabla})^{m} G(\vec{\nabla})^{n} \psi$. One can check that the mixing matrix for local operators corresponding to (13) coincides with eq. (54) in ref. [6] to the stated $1 / N_{c}^{2}$ accuracy.

Here we deviate from the standard procedure and search for the solutions of the evolution equation (12) in the form of nonlocal operators:

$\int_{-1}^{1} \mathrm{~d} \xi \phi_{j}^{ \pm}(\xi) S^{ \pm}(j, \xi)$.

Hence we work with the integral equations conjugate to (14) for the function $\phi_{j}^{ \pm}(\xi)$ :

$\int_{-1}^{1} \mathrm{~d} \eta K_{j}^{ \pm}(\eta, \breve{\zeta}) \phi_{j}^{ \pm}(\eta)=\gamma_{j} \phi_{j}^{ \pm}(\xi)$.

We are able to prove that eqs. (16) have one and only one pair of solutions which are analytic at the points $\xi= \pm 1$ in the limit $j \rightarrow \infty$ :

$\phi^{+}(\xi)=1-\xi, \quad \phi^{-}(\xi)=1+\xi$,

and the corresponding eigenvalue (anomalous dimension) equals

$\gamma_{j}^{\mathrm{NS}}=2 N_{\mathrm{c}}\left(\psi(j+1)+\gamma_{\mathrm{E}}-\frac{1}{4}+\frac{1}{2(j+1)}\right)$

where $\psi(z)=(\mathrm{d} / \mathrm{d} z) \ln \Gamma(z)$ and $\gamma_{\mathrm{E}}$ is the Euler constant. The validity of eqs. (17) and (18) can easily be checked by a straightforward calculation.

Inserting eq. (17) in (15) and comparing with (11) we see that precisely these nonlocal operators contribute at the tree level to the structure function $g_{2}^{\mathrm{tw} .3}\left(x, Q^{2}\right)$. Thus, we arrive at eq. (3) with the anomalous dimension (18). All other operators decouple from $g_{2}^{\text {tw.3 }}\left(x, Q^{2}\right)$ in the $N_{c} \rightarrow \infty$ limit.

For integer values of $j=n$ we can go over to local operators. In the notations of ref. [6] the solution (15) (with $\phi^{ \pm}$given in (17)) becomes

$(n+1) \cdot \frac{1}{2} \int_{-1}^{!} \mathrm{d} \xi\left[\phi^{+}(\xi) S^{+}(n, \xi)+\phi^{-}(\xi) S^{-}(n, \xi)\right]=\sum_{k=1}^{n-1}(n-k) Y_{n}^{k}$.

By comparison with the numerical calculations of the spectrum shown in fig. 2, we find that the analytic expres-

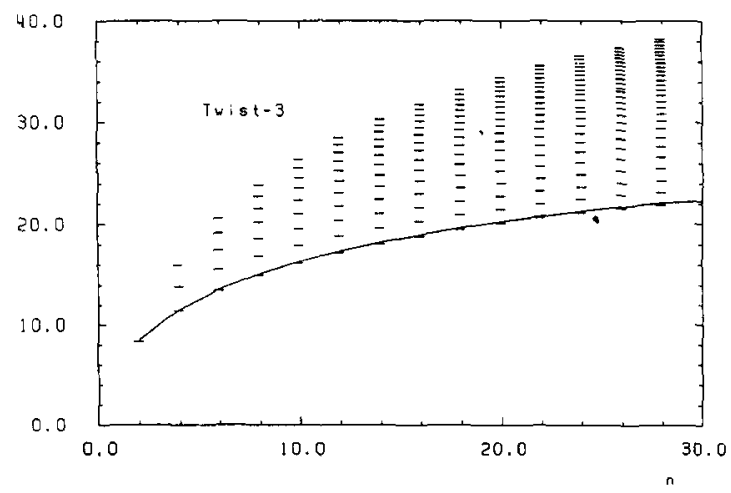

Fig. 2. The same as in fig. 1, but with the substitution $C_{\mathrm{f}}=\frac{1}{2} N_{\mathrm{c}}$, which corresponds to the limit of a large number of colours, compared to the analytic solution (18). 
sion (18) reproduces the lowest anomalous dimensions of the quark-gluon operators for each moment. For example, for the second moment $j=2$ we obtain from (18) $\gamma_{2}^{\mathrm{NS}}=\frac{17}{6} N_{\mathrm{c}}$, in agreement with ref. [5]. For the fourth moment one obtains $\gamma_{4}^{\mathrm{NS}}=\frac{58}{15} N_{\mathrm{c}}$, which coincides with the lowest of anomalous dimensions found in ref. [9]. It is easy to check that a small difference is indeed the $1 / N_{\mathrm{c}}^{2}$ effect. Taking all the $1 / N_{\mathrm{c}}^{2}$ corrections into account we obtain by explicit diagonalization of the mixing matrix from [6]

$$
\begin{aligned}
& \frac{5}{2} \int_{0}^{1} \mathrm{~d} x x^{4} g_{2}^{\text {w. } .3}\left(x, Q^{2}\right)=\left[0 . 8 1 \left\langle\left\langle Y_{4}^{3}\left(\mu^{2}\right)\right\rangle+2.32\left\langle\left\langle Y_{4}^{2}\left(\mu^{2}\right)\right\rangle+2.89\left\langle\left\langle Y_{4}^{1}\left(\mu^{2}\right)\right\rangle\right]\left(\frac{\alpha_{\mathrm{s}}\left(Q^{2}\right)}{\alpha_{\mathrm{s}}\left(\mu^{2}\right)}\right)^{\gamma_{1} / b}\right.\right.\right. \\
& \quad+\left[0 . 0 2 6 \left\langle\left\langle Y_{4}^{3}\left(\mu^{2}\right)\right\rangle+0.013\left\langle\left\langle Y_{4}^{2}\left(\mu^{2}\right)\right\rangle-0.024\left\langle\left\langle Y_{4}^{1}\left(\mu^{2}\right)\right\rangle\right]\left(\frac{\alpha_{\mathrm{s}}\left(Q^{2}\right)}{\alpha_{\mathrm{s}}\left(\mu^{2}\right)}\right)^{\gamma_{2} / b}\right.\right.\right. \\
& \quad+\left[0 . 1 6 \left\langle\left\langle Y_{4}^{3}\left(\mu^{2}\right)\right\rangle-0.33\left\langle\left\langle Y_{4}^{2}\left(\mu^{2}\right)\right\rangle+0.13\left\langle\left\langle Y_{4}^{1}\left(\mu^{2}\right)\right\rangle\right]\left(\frac{\alpha_{\mathrm{s}}\left(Q^{2}\right)}{\alpha_{\mathrm{s}}\left(\mu^{2}\right)}\right)^{\gamma_{3} / b}\right.\right.\right. \\
& \quad \gamma_{1}=10.89, \quad \gamma_{2}=13.71, \quad \gamma_{3}=16.15 .
\end{aligned}
$$

It is seen that the contributions of the operators with higher anomalous dimensions are indeed small and that the coefficients in front of the three terms with the lowest anomalous dimension are close to their values in the $N_{\mathrm{c}} \rightarrow \infty$ limit, 1, 2, and 3, respectively. We have checked that neglecting the $1 / N_{\mathrm{c}}^{2}$ terms in the mixing matrix of ref. [6] we arrive precisely at eq. (3).

The result (20) disagrees, however, with the expression given in ref. [9], although the anomalous dimensions coincide. An inspection suggests that the difference between the results of refs. [6,8] and ref. [9] is due to an overall sign of the contributions of operators containing the $\gamma_{s}$ matrix. In our language the effect would be in the interchange of the operators $S^{+}$and $S^{-}$in eq. (11). The statement that in the $N_{\mathrm{c}} \rightarrow \infty$ limit the operators with lowest anomalous dimensions decouple from the evolution equation remains, in any case, true.

\section{3. $Q^{2}$-evolution of $g_{2}\left(x, Q^{2}\right)$ in the $j \rightarrow \infty$ limit}

It is well known that the behaviour of structure functions in the region $x \rightarrow 1$ is determined by the asymptotics of the corresponding anomalous dimensions in the $j \rightarrow \infty$ limit. In this limit all $1 / N_{\mathrm{c}}^{2}$ corrections to the kernel in eq. (13) are reduced to the factors $C_{\mathrm{f}} / N_{\mathrm{c}}$ which can easily be read off the complete expression given in ref. [8]. We arrive at the following integral equation:

$\gamma_{j} \phi(\xi)=4 C_{\mathrm{f}}\left(\ln j+\gamma_{\mathrm{E}}-\frac{3}{4}\right) \phi(\xi)+N_{\mathrm{c}} \phi(\xi)+N_{\mathrm{c}} \int_{-1}^{\xi} \mathrm{d} \eta\left(\frac{1-\xi}{1-\eta}\right)^{2} \frac{\phi(\xi)-\phi(\eta)}{\xi-\eta}+N_{\mathrm{c}} \int_{\xi}^{1} \mathrm{~d} \eta\left(\frac{1+\xi}{1+\eta}\right)^{2} \frac{\phi(\xi)-\phi(\eta)}{\eta-\xi}$.

Note that in this limit, the kernels $K^{+}$and $K^{-}$coincide and hence we drop the superscripts ( \pm ) from $K^{ \pm}$and $\phi^{ \pm}$. The kernel in $(21)$ becomes symmetric after multiplication by $1 /\left(1-\xi^{2}\right)^{2}$, so that the eigenfunctions should be orthogonal with this weight function. Thus, the solutions which are analytic at the points $\xi= \pm 1$ are given by the Gegenbauer polynomials with a negative superscript $C_{n}^{-3 / 2}(\xi)$. They exist for $n=0$ and $n=1$ only and do not form a complete set of functions. We end up with the two solutions given in (17), which both satisfy eq. (21), corresponding to the same anomalous dimension

$\gamma_{j}^{\mathrm{NS}}=4 C_{\mathrm{f}}\left(\ln j+\gamma_{\mathrm{E}}-\frac{3}{4}\right)+N_{\mathrm{c}}$.

\#2 The particular expression for the mixing matrix for $n=4$ in ref. [6] contains misprints which can be figured out by comparing it to the general expression eq. (54) in this reference or to later publications by the same authors [12]. Our thanks are due to A.P. Bukhvostov for correspondence on this point. 
We argue that these solutions satisfy the complete evolution equation to the $(1 / j) \ln j$ accuracy. This issue is not trivial. To illustrate the difficulty involved let us return to the evolution equation in the $N_{\mathrm{c}} \rightarrow \infty$ limit, discussed in the previous section. We easily find that each of the functions (17) provides an approximate solution to the integral equation with the counterpart kernel

$\frac{1}{N_{\mathrm{c}}} \int_{-1}^{1} \mathrm{~d} \eta K^{ \pm}(\eta, \xi) \phi^{\mp}(\eta)=2\left[\psi(j+1)+\gamma_{\mathrm{E}}-\frac{1}{4}\right] \phi^{\mp}(\xi)-\frac{2}{j+1} \phi^{ \pm}(\xi)+2 \int_{0}^{1} \frac{\mathrm{d} \theta}{\theta+(1 \mp \xi) /(1 \pm \xi)}(1-\theta)^{j}$.

All the terms on the RHS of (23) except for the first one are down by the factor $1 / j$. However, a simple expansion of the last term in a power series,

$\int_{0}^{1} \frac{\mathrm{d} \theta}{\theta+(1 \mp \xi) /(1 \pm \xi)}(1-\theta)^{j}=\frac{1}{j} \frac{1 \pm \xi}{1 \mp \xi}+\mathrm{O}\left(\frac{1}{j^{2}}\right)$,

would introduce dangerous singularities at $\xi= \pm 1$, giving rise to logarithmic divergences in the matrix elements of the corresponding nonlocal operators $(15)$. Thus the mixing is actually of the order of $(1 / j) \ln j$. In the language of local operators the origin of this difficulty can be explained as follows. Eq. (23) states that the coefficient in the mixing matrix in front of the particular local operator corresponding to $\phi^{\mp}(\xi)=1 \pm \xi$ is of the order of unity, while all mixing coefficients are down by $1 / j$. However, since the total number of operators is large, of the order of $j$, the danger is that the smallness of the coefficients can be compensated by a large number of contributions. The increase is actually of the order of $\ln j$ only, which follows readily from (23) under the assumption that the matrix elements of the operators $S^{ \pm}(\xi)$ are nonsingular at $\xi= \pm 1$. Including the $1 / N_{\mathrm{c}}$ corrections does not bring in any principle complications. We substitute (17) in the exact evolution equation and make sure that retaining the $\mathrm{O}(1 / j)$ corrections does not bring in mixing with functions which contain singularities stronger than linear at the end points $\xi= \pm 1$. Corresponding matrix elements are then of the order of $\ln j$.

Comparing eq. (22) with the well known result for the asymptotics of anomalous dimensions of operators of twist $2, \gamma_{j}^{\mathrm{tw}-2}=4 \mathrm{C}_{j}\left(\ln j+\gamma_{\mathrm{E}}-\frac{3}{4}\right)+\ldots$, we find that the coefficients in front of $\ln j$ turn out to be equal while the constant term for the twist-3 operators is larger by $N_{\text {c. }}$.

Physical interpretation of this result becomes quite transparent if one remembers that the particular combination of the quark-gluon operators (7) which enters $g_{2}^{\text {tw.3 }}\left(x, Q^{2}\right)$ coincides with the quark operator (1), and the difference to the leadingtwist is in specific spin projection only. It is well known [13] that the logarithmic rise of anomalous dimensions in QCD is due to the emission of soft gluons and that the factor in front of $\ln j$ is determined entirely by the colour charge of the parton (quark). On the other hand, the constant term is affected by the emission of hard gluons and does depend on the spin state of the parent quark.

From eq. (22) it follows readily that in the limit $1-x \ll 1$

$g_{2}^{\mathrm{NS}}\left(x, Q^{2}\right) \sim F\left(Q^{2}\right)(1-x)^{\left(4 C_{\mathrm{r}} / b\right) \ln \left[\alpha_{\mathrm{s}}\left(\mu^{2}\right) / \alpha_{\mathrm{s}}\left(Q^{2}\right)\right]-1}\left[1+T\left(\mu^{2}\right)\left(\frac{\alpha_{\mathrm{s}}\left(Q^{2}\right)}{\alpha_{\mathrm{s}}\left(\mu^{2}\right)}\right)^{N_{\mathrm{c}} / b}\right]$,

where $F\left(Q^{2}\right)$ is a $Q^{2}$-dependent normalization factor, determined by the twist-2 contribution to $g_{2}$, and the term $T\left(\mu^{2}\right)$ stands for relative contributions of twist-3. On the evidence of various model estimations we expect it to be negative. In other words, twist three contributions to $g_{2}\left(x, Q^{2}\right)$ have the same power behaviour at $1-x \ll 1$ as terms of the leading twist and fall off slowly with increasing $Q^{2}$.

The same conclusion can be reached in the flavour singlet channel for contributions of gluons. We are able to calculate in a similar way the asymptotics of anomalous dimensions of the relevant nonlocal three-gluon operator:

$G_{\mu}(\alpha, \beta, \gamma)=\frac{1}{2} f_{a b c} z_{p} G_{\xi}^{a}(\alpha z) z_{\sigma} \widetilde{G}_{\mu \sigma}^{b}(\beta z) z_{\eta} G_{\xi \eta}^{c}(\gamma z)$.

Making the Mellin transformation (10) and searching for a multiplicatively renormalized nonlocal three-gluon 


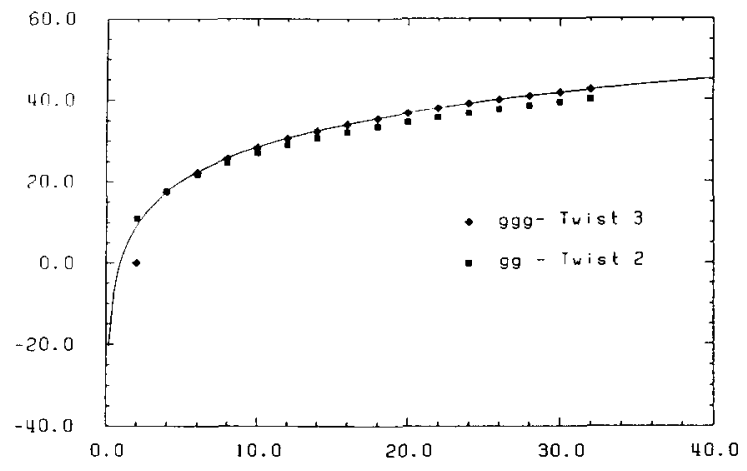

Fig. 3. Anomalous dimensions of the three-gluon operators of twist-3 (with $n_{\mathrm{f}}=3$ ) neglecting the mixing with the quark operators (shown by solid diamonds), compared to the anomalous dimensions of two-gluon operators of twist 2 (shown by solid squares) and to the asymptotic formula (28).

operator in a form analogous to (16), we arrive at an integral equation for the corresponding weight function $\phi_{j}^{\mathrm{G}}(\xi)$ :

$$
\begin{aligned}
& \gamma_{j}^{\mathrm{GGG}} \phi^{\mathrm{G}}(\xi)=4 N_{\mathrm{c}}\left(\ln j+\gamma_{\mathrm{E}}+\frac{1}{4}\right) \phi^{\mathrm{G}}(\xi)-b \phi^{\mathrm{G}}(\xi) \\
& \quad+N_{\mathrm{c}} \int_{-1}^{\xi} \mathrm{d} \eta\left(\frac{1-\xi}{1-\eta}\right)^{2} \frac{\phi^{\mathrm{G}}(\xi)-\phi^{\mathrm{G}}(\eta)}{\xi-\eta}+N_{\mathrm{c}} \int_{\xi}^{1} \mathrm{~d} \eta\left(\frac{1+\xi}{1+\eta}\right)^{2} \frac{\phi^{\mathrm{G}}(\xi)-\phi^{\mathrm{G}}(\eta)}{\eta-\xi} .
\end{aligned}
$$

Eq. (27) follows readily from eq. (6.9) in ref. [8] neglecting all $\sim 1 / j$ terms, and differs from (21) by trivial factors only. Hence, we get the same pair of solutions $\phi^{\mathrm{G}}(\xi)=1 \pm \xi$. The anomalous dimension in this case is

$\gamma_{j}^{\mathrm{GGG}}=4 N_{\mathrm{c}}\left(\ln j+\gamma_{\mathrm{E}}+\frac{1}{4}\right)-b$,

and exceeds the asymptotics of anomalous dimensions of two-gluon operators of twist- 2 by the same constant $N_{\mathrm{c}}, \gamma_{j}^{\mathrm{GG}}=4 N_{\mathrm{c}}\left(\ln j+\gamma_{\mathrm{E}}\right)-b+\ldots$. Thus, the difference in the behaviour of twist-2 and -3 gluon distributions at large $-x$ is the same as that for the quark ones. The asymptotics formula (28) is compared with the results of numerical calculations of the spectrum of anomalous dimensions in fig. 3 .

\section{Concluding remarks}

We have found an explicit solution of the evolution equation for $g_{2}^{\text {tw. } 3}\left(x, Q^{2}\right)$ in the large- $N_{\mathrm{c}}$ limit which gives rise to the series of local operators with the lowest anomalous dimension. To proceed further, one may try to find a general solution of the asymptotic equation (21) which could provide a basis of functions in search of multiplicatively renormalized nonlocal operators. At least $\mathrm{O}(1 / j)$ corrections to anomalous dimensions and the operator mixing can be studied in this way. An inspection of (21) shows that these solutions should possess logarithmic branching points at $\xi= \pm 1$ and be orthogonal with the weight function $1 /\left(1-\xi^{2}\right)^{2}$, which suggests Legendre functions $Q(z)$ as a natural candidate. This work is in progress.

Including all the $1 / N_{\mathrm{c}}$ corrections to the evolution equation for nonsinglet operators and studying evolution of gluon operators beyond the $j \rightarrow \infty$ limit seems to be more difficult. The point is that in the expressions discussed above we have had a very important simplification as compared to the general case, in that the evolution kernels have been vanishing for contributions with the interchanged ordering of partons on the light-cone. It can be traced that this property has allowed us to search for the nonlocal solutions (15) of the evolution equation with the support property $-1<\xi<1$. In general situations, the integration regions are stretched up to infinity and a nontrivial analytical structure arises at $\xi= \pm 1$.

Using explicit expressions for the mixing matrices of local operators from ref. [6] we have carried out extensive numerical calculations of the spectrum of anomalous dimensions for both the flavour-nonsinglet and 
-singlet operators. Some of the results are shown in figs. 1-3, where comparisons with the analytic results obtained in the stated limits are also shown. We give below a parametrization for the lowest anomalous dimension in the spectrum of nonsinglet operators, which is valid within $0.1 \%$ accuracy for $j \geqslant 2$, and which has the correct behaviour for the large- $N_{\mathrm{c}}$ and large- $n$ limits:

$\gamma_{j}^{\mathrm{NS}, \min }=4 C_{\mathrm{f}}\left(1+\frac{1}{2}+\ldots+\frac{1}{j}-\frac{3}{4}\right)+N_{\mathrm{c}}\left(1+\frac{1}{j+1}\right)+\frac{1}{N_{\mathrm{c}}} \frac{10}{j(j+1)}$.

In the region $2 \leqslant j \leqslant 32$ the splitting between the lowest and the second lowest anomalous dimensions in the spectrum can be approximated by the formula

$\delta \gamma_{j}^{\mathrm{NS}, \min }=4 C_{\mathrm{f}}(0.21+1.29 / j)$.

In the same interval of $j$ the difference between the lowest and the largest anomalous dimensions behaves as

$\gamma_{j}^{\mathrm{NS} \cdot \max }-\gamma_{j}^{\mathrm{NS} \cdot \min }=4 C_{\mathrm{f}}(1.175 \ln j-0.491-0.642 / j)$.

The difference in the anomalous dimensions for the flavour-singlet and flavour-nonsinglet operators is distinguishable for $j=2$ and $j=4$ only. Complete results will be published elsewhere.

\section{Acknowledgement}

We are grateful to I.I. Balitsky and A.P. Bukhvostov for correspondence. One of us (V.B.) would like to thank the DESY theory group for its hospitality. He would also like to thank Bo Andersson and participants of the QCD Workshop at Lund, where a part of this work has been done, for hospitality and stimulating discussions.

\section{References}

[1] EM Collab., J. Ashman et al., Phys. Lett. B 206 ( 1988) 364; CERN preprint CERN-EP/89-73 (1989); V.W. Hughes et al., Phys. Lett. B 212 (1988) 511.

[2] G.G. Ross, in: Proc. 1989 Intern. Symp. on Lepton and photon interactions at high energies (Stanford University, Stanford, CA, USA) (World Scientific, Singapore, 1990) p. 41.

[3] S. Wandzura and F. Wilczek, Phys. Lett. B 82 (1977) 195.

[4] M.A. Ahmed and G.G. Ross, Nucl. Phys. B 111 (1976) 441;

J. Kodaira et al., Phys. Rev. D 20 (1979) 627; Nucl. Phys. B 159 (1979) 99

[5] E.V. Shuryak and A.I. Vainshtein, Nucl. Phys. B 199 (1982) 951.

[6] A.P. Bukhvostov, E.A. Kuraev and L.N. Lipatov, JETP Lett. 37 (1983) 482; Sov. Phys. JETP 60 (1984) 22.

[7] P.G. Ratcliffe, Nucl. Phys. B 264 (1986) 493.

[8] I.I. Balitsky and V.M. Braun, Nucl. Phys. B 311 (1988/89) 541.

[9] X. Ji and C. Chou, Phys. Rev. D 42 (1990) 3637.

[10] V.N. Gribov and L.N. Lipatov, Sov. J. Nucl. Phys. 15 (1972) 78; G. Altarelli and G. Parisi, Nucl. Phys. B 126 (1977) 298.

[11] 1.I. Balitsky and V.M. Braun, in: Proc. XXV LNPI Winter School on Physics (Leningrad, 1990) pp. 105-153.

[12] A.P. Bukhvostov, E.A. Kuraev and L.N. Lipatov, Sov. J. Nucl. Phys. 39 (1984) 121; Nucl. Phys. B 258 (1985) 601.

[13] Yu.L. Dokshitzer, D.I. Dyakonov and S.I. Troyan, Phys. Rep. 58 (1980) 269. 